${ }^{1}$ Division of Pulmonary and Sleep Medicine, Children's National Medical Center, Washington, DC, USA. ${ }^{2}$ Dept of Pediatrics, George Washington University School of Medicine and Health Sciences, Washington, DC, USA. ${ }^{3}$ Dept of Integrative Systems Biology, George Washington University, Washington, DC, USA. ${ }^{4}$ Center for Genetic Research Medicine, Children's National Medical Center, Washington, DC, USA. ${ }^{5}$ Division of Pediatric Otorhinolaryngology, Depts of Surgery and Pediatrics, George Washington University School of Medicine and Health Sciences, Washington, DC, USA. 'Division of Emergency Medicine, Dept of Pediatrics, George Washington University School of Medicine and Health Sciences, Washington, DC, USA. ${ }^{7}$ Dept of Biochemistry and Molecular Medicine, George Washington University, Washington, DC, USA.

Correspondence: Gustavo Nino, Division of Pediatric Pulmonology and Sleep Medicine, Children's National Medical Center, Center for Genetic Medicine Research, 111 Michigan Avenue, NW, Washington, DC 20010, USA.

E-mail: gnino@childrensnational.org

Received: March 132014 | Accepted after revision: May 122014 | First published online: June 252014

Support statement: This study was supported by NIH grants HL090020, HD001399 and the Board of Visitors (BOV) grant of Children's National Medical Center.

Conflict of interest: None declared.

\title{
References
}

Lemanske RF Jr, Jackson DJ, Gangnon RE, et al. Rhinovirus illnesses during infancy predict subsequent childhood wheezing. J Allergy Clin Immunol 2005; 116: 571-577.

2 Schneider D, Hong JY, Popova AP, et al. Neonatal rhinovirus infection induces mucous metaplasia and airways hyperresponsiveness. J Immunol 2012; 188: 2894-2904.

Ziegler SF, Artis D. Sensing the outside world: TSLP regulates barrier immunity. Nat Immunol 2010; 11: 289-293. Mahmutovic-Persson I, Akbarshahi H, Bartlett NW, et al. Inhaled dsRNA and rhinovirus evoke neutrophilic exacerbation and lung expression of thymic stromal lymphopoietin in allergic mice with established experimental asthma. Allergy 2014; 69: 348-358.

5 Kato A, Favoreto S Jr, Avila PC, et al. TLR3- and Th2 cytokine-dependent production of thymic stromal lymphopoietin in human airway epithelial cells. J Immunol 2007; 179: 1080-1087.

6 Ying S, O'Connor B, Ratoff J, et al. Thymic stromal lymphopoietin expression is increased in asthmatic airways and correlates with expression of Th2-attracting chemokines and disease severity. J Immunol 2005; 174: 8183-8190.

7 Uller L, Leino M, Bedke N, et al. Double-stranded RNA induces disproportionate expression of thymic stromal lymphopoietin versus interferon- $\beta$ in bronchial epithelial cells from donors with asthma. Thorax 2010; 65: 626-632.

8 Fuchimoto J, Kojima T, Kobayashi N, et al. Hop water extract inhibits double-stranded RNA-induced thymic stromal lymphopoietin release from human nasal epithelial cells. Am J Rhinol Allergy 2012; 26: 433-438.

9 Nonaka M, Fukumoto A, Ogihara N, et al. Synergistic induction of thymic stromal lymphopoietin by tumor necrosis factor $\alpha$ and Th2 cytokine in nasal polyp fibroblasts. Am J Rhinol Allergy 2010; 24: e14-e18.

10 Cheng DT, Ma C, Niewoehner J, et al. Thymic stromal lymphopoietin receptor blockade reduces allergic inflammation in a cynomolgus monkey model of asthma. J Allergy Clin Immunol 2013; 132: 455-462.

11 Han H, Headley MB, Xu W, et al. Thymic stromal lymphopoietin amplifies the differentiation of alternatively activated macrophages. J Immunol 2013; 190: 904-912.

12 Zhu J, Message SD, Qiu Y, et al. Airway inflammation and illness severity in response to experimental rhinovirus infection in asthma. Chest 2014; 145: 1219-1229.

13 Message SD, Laza-Stanca V, Mallia P, et al. Rhinovirus-induced lower respiratory illness is increased in asthma and related to virus load and Th1/2 cytokine and IL-10 production. Proc Natl Acad Sci USA 2008; 105: 13562-13567.

14 Konstantinou GN, Xepapadaki P, Manousakis E, et al. Assessment of airflow limitation, airway inflammation, and symptoms during virus-induced wheezing episodes in 4- to 6-year-old children. J Allergy Clin Immunol 2013; 131: 87-93.

15 Castro-Rodriguez JA, Molina RO, Cáceres M, et al. Correlation between nitrites in induced sputum and asthma symptoms in asthmatic school children. Pediatr Pulmonol 2014; 49: 214-220.

\section{Increased prevalence of pre-school wheeze is not explained by time trends in body mass index}

To the Editor:

Overweight and obesity have been associated with asthma, particularly in adults [1-4]. In children, findings are less consistent $[2,5-7]$. Different mechanisms have been proposed to explain this association, including lung mechanics, systemic inflammation and associated comorbidities $[1,2,8]$. In a previous study, we 
showed that the prevalence of pre-school wheeze doubled in two cohorts in Leicestershire, UK, born 8 years apart [9]. This increase could not be attributed to differences in environmental exposures, socioeconomic status or vaccination status $[9,10]$. Because body mass index (BMI) is increasing across the globe [2], we hypothesised that BMI played a role. We therefore sought to determine whether higher BMI in the second cohort could explain the increase in prevalence of pre-school wheezing and asthma.

Our data come from the Leicester Respiratory Cohorts, two population-based random samples of children living in Leicestershire [11]. Cohort 1 included children born in 1985-1989; cohort 2 included children born in 1993-1997. At the time the first respiratory questionnaire was administered (1990 and 1998), children were 1-4 years old. An identical study protocol was followed for both cohorts [11]. Parents completed a postal questionnaire that asked for detailed information on respiratory symptoms and environmental exposures. Routine data, including demographic and ethnic information, and data on growth and immunisations, were available from the Leicestershire Health Authority Database. Height and weight had been measured and recorded independently by health visitors. We used the measurements made closest to the date of the questionnaire for analyses. The Leicestershire Health Authority Research Ethics Committee (Leicester, UK) approved the study.

We included white pre-school children aged 1-4.99 years, with completed questionnaires and at least one valid measurement of height and weight (table 1) [9, 11]. BMI was calculated from growth measurements, as weight in kilograms divided by height in meters squared. We used z-scores from the World Health Organization (WHO) growth standards as reference values for BMI [12]. We also computed BMI categories (underweight, normal weight, overweight and obese) from WHO [12] to investigate whether outcomes were associated with extreme values (overweight, obese and underweight) rather than a linear trend.

Main outcomes were prevalence of current wheeze (previous 12 months) and doctor-diagnosed asthma. Secondary outcomes were frequent wheeze (three or more episodes in previous 12 months), multipletrigger wheeze and hospital admission for wheeze (ever in life).

We calculated odds ratios for time trends (1998 versus 1990) in current wheeze and doctor-diagnosed asthma with multivariable logistic regression, with and without adjusting for BMI z-scores and BMI categories. We repeated the unadjusted analysis, comparing children with and without valid BMI measurements to check for systematic differences between the groups. Sensitivity analyses were performed for the secondary outcomes. We controlled for various potential confounding factors (sex, age, pets, preand post-natal exposure to smoke, gas cooking, attending nursery care, parental education, and crowding).

In total, we posted 1650 questionnaires to cohort 1, of which 1422 (86\%) were returned. We posted 2522 questionnaires to cohort 2, of which $2127(84 \%)$ were returned. For the analysis, we excluded data from children aged less than 1 and more than 5 years, leaving 1136 children in cohort 1 and 2084 children in cohort 2. In cohort 1, 978 children, and in cohort 2, 1819 children had valid measurements for height and weight.

Between 1990 and 1998, mean BMI z-scores increased from -0.693 (95\% CI -0.143-0.004) to 0.104 (95\% CI $0.052-0.156)(\mathrm{p}<0.001)$ (table 1$)$. In the same period, prevalence of current wheeze rose from $11.9 \%$ to $26.3 \%$ and doctor-diagnosed asthma rose from $11.0 \%$ to $18.6 \%$ (both $\mathrm{p}<0.001$ ) (table 1). Prevalence of secondary outcomes also increased (table 1).

In cohort 1 (1990), there was no association between current wheeze or doctor-diagnosed asthma and BMI $\mathrm{z}$-score $(\mathrm{p}=0.297$ and $\mathrm{p}=0.443$, respectively) or BMI categories. In cohort 2 (1998), current wheeze and doctor-diagnosed asthma were associated with BMI z-scores; the odds ratio was 1.16 for wheeze (95\% CI $1.05-1.27, \mathrm{p}=0.002)$ and 1.13 for asthma $(95 \%$ CI $1.02-1.25, \mathrm{p}=0.025)$ per $1 \mathrm{z}$-score of BMI. When looking at BMI categories, we found that compared to children of normal weight, obese children had odds ratios of 1.57 (95\% CI 1.16-2.12, $\mathrm{p}=0.003)$ for wheeze and $1.68(95 \%$ CI $1.21-2.35, \mathrm{p}=0.002)$ for asthma. Overweight and underweight were not associated with these outcomes $(p=0.337$ and $p=0.282$ for current wheeze, and $\mathrm{p}=0.994$ and $\mathrm{p}=0.815$ for doctor-diagnosed asthma, respectively).

Last, we compared the prevalence of respiratory outcomes between 1990 and 1998. When we compared 1998 to 1990, the unadjusted odds ratios were 2.73 (95\% CI 2.19-3.41) for current wheeze and 1.79 (95\% CI 1.42-2.26) for doctor-diagnosed asthma (fig. 1). We repeated this analysis, comparing children with and without valid BMI measurements, with similar findings (data not shown). The results were unchanged when we adjusted for BMI z-scores (adjusted odd ratios were 2.68 (95\% CI 2.15-3.35) for current wheeze and 1.77 (95\% CI 1.40-2.24) for doctor-diagnosed asthma) and for BMI categories (fig. 1). Adjusting for other potential confounders also did not change the odds ratios. The results of sensitivity analyses for secondary outcomes were similar to primary outcomes (data available from the authors).

In summary, we found: 1) prevalence of reported wheeze and doctor-diagnosed asthma in preschool children increased significantly between 1990 and 1998, and BMI rose over the same period; 2) BMI and 


\section{TABLE 1 Demographic data, body mass index (BMI) and respiratory morbidity in the two cohorts}

Cohort 1

Subjects $n$

Year of questionnaire survey

Age years

Males

BMI z-scores

Current wheeze

Doctor-diagnosed asthma

Frequent wheeze ${ }^{\#}$

Multiple-trigger wheeze

Hospital admission for wheeze
978

1990

$2.99(2.92-3.06)$

50.6 (495) (47.5-53.8)

$-0.693(-0.143-0.004)$

$11.9(116)(9.8-13.9)$

$11.0(108)(9.1-13.0)$

$6.1(60)(4.6-7.6)$

$5.1(50)(3.7-6.5)$

$3.4(33)(2.2-4.5)$
Cohort 2

1819
1998
$3.13(3.08-3.18)$
$52.7(958)(50.4-55.0)$
$0.104(0.052-0.156)$
$26.3(478)(24.3-28.3)$
$18.6(339)(16.8-20.4)$
$13.4(244)(11.8-15.0)$
$9.8(179)(8.5-11.2)$
$6.4(117)(5.3-7.6)$

$52.7(958)(50.4-55.0)$

$26.3(478)(24.3-28.3)$

18.6 (339) (16.8-20.4)

9.8 (179) (8.5-11.2)

$6.4(117)(5.3-7.6)$

Data are presented as mean $(95 \% \mathrm{CI})$ or \% (n) (95\% CI), unless otherwise stated. *: three or more episodes in the previous 12 months; ${ }^{\circ}$ : wheezing occurring also between episodes of respiratory infections.

respiratory outcomes were associated in the more recent cohort but not in the earlier one; and 3) no evidence that increased BMI explains the marked increase in wheeze and asthma prevalence over this 8 -year period.

We believe this is the first study to examine the possibility that BMI explains time trends in wheezing illness in toddlers. CHINN and RoNA [5] conducted a comparable study for schoolchildren with similar findings. They also found that BMI and asthma were associated in obese children only in the more recent cohort (1994) and not in the earlier cohort (1982). Also in their population, rising BMI did not explain trends in respiratory outcomes.

Most other studies examined the relationship between BMI and asthma in a single cohort only, and focused on older children or adults. Associations were not consistent in children and were found mainly for preadolescents $[2,6,7]$. In a birth cohort, NoAL et al. [7] found that obesity and wheezing were associated in 11-year-olds but not in 15-year-olds. GUERRA et al. [6] reported that obesity at age 11 years predicted persistence of wheeze and asthma during puberty.

Our study was strengthened by the use of identical methodology in both cohorts; cohort 2 had been designed to assess time trends [9]. We used objective measurements of weight and height, which were assessed independently from the outcomes. We were, however, limited by parent-reporting of respiratory

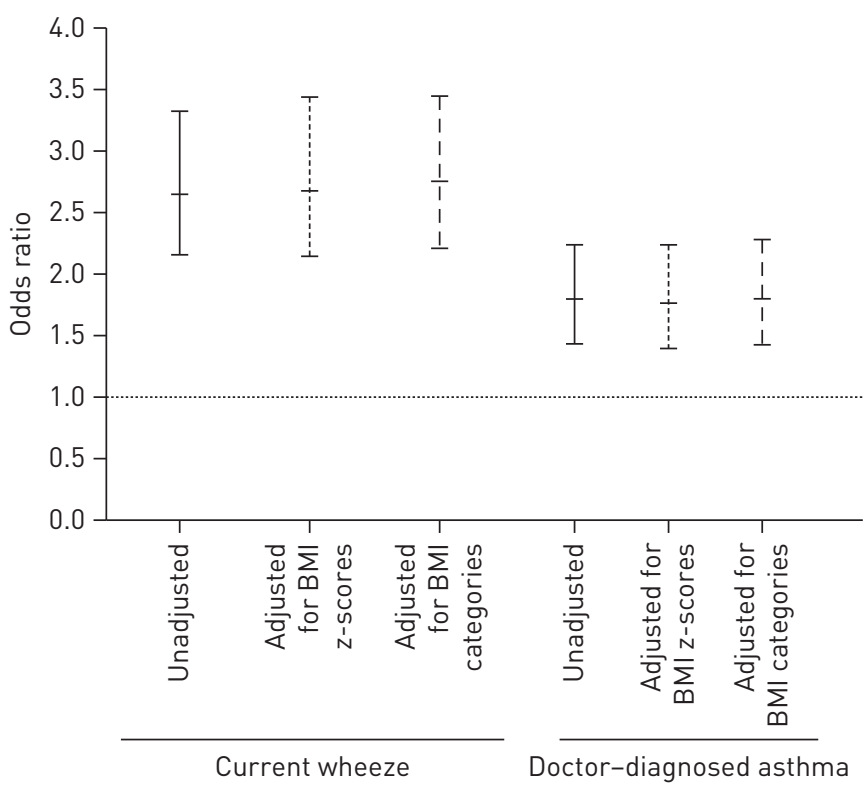

FIGURE 1 Odds ratios and 95\% confidence intervals describing the trend of respiratory outcomes (1998 versus 1990) unadjusted, adjusted for body mass index (BMI) z-scores and adjusted for BMI categories. 
outcomes. We lacked data on maternal adiposity, which has been associated with transient wheeze in the offspring and could be a confounder [13]. As with all questionnaire-based surveys, a non-response bias could have affected our results. However, our response rate was high (84-86\%) so our sample remains highly representative. BMI could not be calculated in $13 \%$, because measurements of height and weight had not been taken on the same date, but missingness was not associated with the outcome and can hardly have affected the results.

If BMI is associated with wheeze or asthma, the connection is recent, because it was only seen in our second cohort. This could possibly be explained by lifestyle factors that differ between obese children and children of normal weight, and have emerged only recently, such as differences in diet, exercise habits or time spent indoors. The lack of consistency in the relationship between BMI and wheeze over time, also observed by Chinn and Rona [5], makes a causal association less likely. Our data do not exclude, however, that the association between BMI and wheeze may be relevant for very obese children, or for adults and adolescents $[7,14]$. Neither do they contradict an association of birth weight and weight gain during early childhood with childhood asthma, as found in a recent meta-analysis including data from the Leicester cohorts [15]. Finally, BMI might be associated with specific phenotypes of wheezing illness only. In summary, our study suggests that the generational increase in BMI does not explain the increasing prevalence of wheezing in Leicestershire pre-school children throughout the 1990s.

Generational increase in BMI cannot explain increasing prevalence of wheezing in preschool children in the 1990s http://ow.ly/xc0Yw

Myrofora Goutaki ${ }^{1}$, Anina M. Pescatore ${ }^{1}$, Parvinder Singh ${ }^{1}$, Caroline S. Beardsmore ${ }^{2}$ and Claudia E. Kuehni ${ }^{1}$ ${ }^{1}$ Institute of Social and Preventive Medicine, University of Bern, Bern, Switzerland. ${ }^{2}$ Dept of Infection, Immunity and Inflammation, University of Leicester, Leicester, UK.

Correspondence: Claudia E. Kuehni, Institute of Social and Preventive Medicine, University of Bern, Finkenhubelweg 11, CH-3012 Bern, Switzerland. E-mail: kuehni@ispm.unibe.ch

Received: Jan 152014 | Accepted after revision: May 202014 | First published online: June 252014

Support statement: The Swiss National Science Foundation (grants 3200B0-122341, 3200B0-144068 and PDFMP3123162) and Asthma UK (grant 07/048) funded this study.

Conflict of interest: Disclosures can be found alongside the online version of this article at www.erj.ersjournals.com

Acknowledgements: We thank the parents of the Leicestershire respiratory cohort children for completing the questionnaires. We thank Cristian Dogaru (Institute of Social and Preventive Medicine, University of Bern, Bern, Switzerland, and School of Applied Social Sciences, University Campus Suffolk, Ipswich, UK) and Ben Spycher (Institute of Social and Preventive Medicine) for their contribution to the statistical analyses, and we thank Kali Tal (Institute of Social and Preventive Medicine) for her editorial assistance.

\section{References}

Beuther DA, Weiss ST, Sutherland ER. Obesity and asthma. Am J Respir Crit Care Med 2006; 174: 112-119.

Boulet LP. Asthma and obesity. Clin Exp Allergy 2013; 43: 8-21.

Beuther DA, Sutherland ER. Overweight, obesity, and incident asthma: a meta-analysis of prospective epidemiologic studies. Am J Respir Crit Care Med 2007; 175: 661-666.

Ford ES. The epidemiology of obesity and asthma. J Allergy Clin Immunol 2005; 115: 897-909.

5 Chinn S, Rona RJ. Can the increase in body mass index explain the rising trend in asthma in children? Thorax 2001; 56: $845-850$.

6 Guerra S, Wright AL, Morgan WJ, et al. Persistence of asthma symptoms during adolescence: role of obesity and age at the onset of puberty. Am J Respir Crit Care Med 2004; 170: 78-85.

$7 \quad$ Noal RB, Menezes AM, Macedo SE, et al. Is obesity a risk factor for wheezing among adolescents? A prospective study in southern Brazil. J Adolesc Health, 2012; 51: Suppl., S38-S45.

8 Beuther DA. Recent insight into obesity and asthma. Curr Opin Pulm Med 2010; 16: 64-70.

9 Kuehni CE, Davis A, Brooke AM, et al. Are all wheezing disorders in very young (preschool) children increasing in prevalence? Lancet 2001; 357: 1821-1825.

10 Kuehni CE, Brooke AM, Davis A, et al. Vaccinations as risk factors for wheezing disorders. Lancet 2001; $358: 1186$.

11 Kuehni CE, Brooke AM, Strippoli MP, et al. Cohort profile: the Leicester respiratory cohorts. Int J Epidemiol 2007; 36: 977-985.

12 De Onis M, Onyango A, Borghi E, et al. WHO Child Growth Standards: Length/height-for-age, weight-for-age, weight-for-length, weight-for-height and body mass index-for-age - Methods and development. Geneva, World Health Organization, 2006.

13 Pike KC, Inskip HM, Robinson SM, et al. The relationship between maternal adiposity and infant weight gain, and childhood wheeze and atopy. Thorax 2013; 68: 372-379. 


\section{RA autoantibodies as predictors of rheumatoid arthritis in non-cystic fibrosis bronchiectasis patients}

To the Editor:

The mechanisms underlying the strong association between rheumatoid arthritis (RA) and bronchiectasis were recently reviewed [1]. A literature review highlighted 289 reports of bronchiectasis associated with RA, with the respiratory symptoms preceding joint symptoms in $90 \%$ of the reports [2], strongly suggesting that the processes in bronchiectasis predispose to RA.

Rheumatoid factor (RF) and anti-cyclic citrullinated antibody (anti-CCP) are integral in the initiation of RA. A Danish study $(n=9712)$ observed that the baseline IgM RF was predictive of a significant, six-fold increased risk of RA development if the RF was two- to four-fold above the normal range [3]. Likewise, a strongly positive anti-CCP test significantly increases the risk of RA (OR 25) [4].

Two studies have investigated RF in bronchiectasis $[5,6]$ with no studies to date investigating anti-CCP. In our BRAC RA (Bronchiectasis, Asthma, Control, Rheumatoid Arthritis) study, a prospective, multicentre, case-control, observational study was conducted to determine the relationship between bronchiectasis and RF and anti-CCP. Recruitment was completed over 12 months using identical methodology and reviewed by the same researcher (E. Perry) with full ethical approval (Integrated Research Application System approval number 12324; Health Research Authority, London, UK). All recruited bronchiectasis patients were under respiratory specialist care, and had high-resolution computed tomography (HRCT) evidence of bronchiectasis and a history of two or more respiratory infections per year. Key exclusion criteria included inflammatory arthritis, tuberculosis or other forms of lung disease. Cystic fibrosis was excluded using genotyping and sweat testing following British Thoracic Society guidelines. Asthma patients were identified from a database where the diagnosis had been confirmed by expert review. Healthy controls were matched with the asthma patients, where possible, for smoking history, age and sex. Anti-CCP measurement was undertaken by ELISA assays including the EliA CCP second-generation ELISA kit (Phadia, Uppsala, Sweden) and IgM RF was quantified using a Modular P analyser (Hitachi, Maidenhead, UK) with cut-off levels as per the manufacturer's instructions. As per the 2010 American College of Rheumatology (ACR)/ European League Against Rheumatism (EULAR) criteria for RA [7], a negative result was defined as within the normal range, a "weakly positive" result as greater than the upper range of normal but below the level of three times the upper range of normal, and a "strongly positive" result as greater than three-fold higher than the upper range of normal. All individuals underwent analysis for RF and anti-CCP. Individuals who developed RA according to the 2010 ACR/EULAR criteria over 12 months were noted [7].

In total 122 bronchiectasis patients, 87 asthma patients and 78 controls were studied. RF positivity was significantly more prevalent in bronchiectasis compared with controls (31 (25.4\%) out of 122 versus eight $(10.3 \%)$ out of $78, \mathrm{p}=0.01)$ but not significantly different when compared with the asthma patients (14 $(16.1 \%)$ out of $87, p=0.13)$. Of those $31 \mathrm{RF}$-positive bronchiectasis patients, four (13\%) were strongly antiCCP positive, versus none of the $91 \mathrm{RF}-$ negative bronchiectasis patients $(\mathrm{p}=0.0036)$. A strongly positive anti-CCP occurred significantly more frequently in bronchiectasis patients (four (3.3\%) out of 122 versus none out of 164 of the asthma and control cases combined, $p=0.03$ by Fisher's exact probability test). Of these four bronchiectasis patients strongly positive for anti-CCP, two subsequently developed RA within 12 months. The demographic details of the bronchiectasis patients, asthma patients and controls are summarised in figure 1 a.

Undoubtedly, a positive RF and anti-CCP in non-RA individuals greatly increases the risk of RA development $[3,4]$. Therefore, our findings of a significantly more prevalent positive RF and anti-CCP in the bronchiectasis patients may explain the reported relationship between bronchiectasis and RA 